\title{
TRIANGULAR UHF ALGEBRAS OVER ARBITRARY FIELDS
}

\author{
R. L. BAKER
}

(Communicated by Palle E. T. Jorgensen)

\begin{abstract}
Let $K$ be an arbitrary field. Let $\left(q_{n}\right)$ be a sequence of positive integers, and let there be given a family $\left\{\Psi_{n m} \mid n \geq m\right\}$ of unital $K$ monomorphisms $\Psi_{n m}: T_{q_{m}}(K) \rightarrow T_{q_{n}}(K)$ such that $\Psi_{n p} \Psi_{p m}=\Psi_{n m}$ whenever $m \leq n$, where $T_{q_{n}}(K)$ is the $K$-algebra of all $q_{n} \times q_{n}$ upper triangular matrices over $K$. A triangular $U H F(T U H F) \quad K$-algebra is any $K$ algebra that is $K$-isomorphic to an algebraic inductive limit of the form $\mathscr{T}=$ $\underset{\lim }{\longrightarrow}\left(T_{q_{n}}(K) ; \Psi_{n m}\right)$. The first result of the paper is that if the embeddings $\Psi_{n m}$

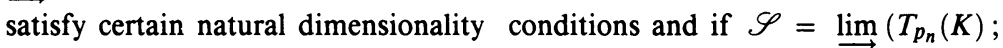
$\left.\Phi_{n m}\right)$ is an arbitrary TUHF $K$-algebra, then $\mathscr{S}$ is $K$-isomorphic to $\mathscr{T}$, only if the supernatural number, $N\left[\left(p_{n}\right)\right]$, of $\left(q_{n}\right)$ is less than or equal to the supernatural number, $N\left[\left(p_{n}\right)\right]$, of $\left(p_{n}\right)$. Thus, if the embeddings $\Phi_{n m}$ also satisfy the above dimensionality conditions, then $\mathscr{S}$ is $K$-isomorphic to $\mathscr{T}$, only if $N\left[\left(p_{n}\right)\right]=N\left[\left(q_{n}\right)\right]$. The second result of the paper is a nontrivial "triangular" version of the fact that if $p, q$ are positive integers, then there exists a unital $K$-monomorphism $\Phi: M_{q}(K) \rightarrow M_{p}(K)$, only if $q \mid p$. The first result of the paper depends directly on the second result.
\end{abstract}

\section{INTRODUCTION}

Let $K$ be a fixed but arbitrary field. In this paper we investigate a certain class of $K$-algebras (i.e., algebras over $K$ ) called triangular $U H F(T U H F) K$ algebras. The main result of our investigation is that for a broad family of TUHF $K$-algebras, the supernatural number associated with these $K$-algebras can be used to compare the isomorphism classes of these $K$-algebras. The main results are stated in Theorem I and Lemma I. For historical remarks on the origins of the idea of a TUHF $K$-algebra, see the remarks following Lemma $I$. In order to present the details of Theorem I and Lemma I, we need the following definitions.

Definition 1.1. Let $K$ be an arbitrary field. Let $\mathscr{A}$ be a unital $K$-algebra. A family of elements $\left\{e_{i j} \mid 1 \leq i \leq j \leq n\right\}$ in $\mathscr{A}$ is said to be a triangular system of algebraic matrix units in $\mathscr{A}$ if, for $1 \leq i \leq j \leq n$, the following conditions are satisfied:

(i) $e_{i j} \neq 0$;

Received by the editors November 23, 1992 and, in revised form, April 19, 1993.

1991 Mathematics Subject Classification. Primary 15A99, 16D70, 46M40; Secondary 47D30.

Key words and phrases. Triangular UHF $K$-algebras, $K$-algebras, inductive limits.

This work was supported by the National Science Foundation. 
(ii) $\left\{e_{i i} \mid 1 \leq i \leq n\right\}$ is an orthogonal family of idempotents such that $\sum_{i=1}^{n} e_{i i}$ $=1$;

(iii) for $1 \leq i \leq p \leq n$ and $1 \leq q \leq j \leq n$ we have

$$
e_{i p} e_{q j}= \begin{cases}e_{i j}, & \text { if } p=q, \\ 0 & \text { otherwise } .\end{cases}
$$

Definition 1.2. Let $\left(p_{n}\right)$ be a sequence of positive integers, and let there be given a family $\left\{\Phi_{n m} \mid n \geq m\right\}$ of unital $K$-monomorphisms $\Phi_{n m}: T_{p_{m}}(K) \rightarrow$ $T_{p_{n}}(K)$ such that $\Phi_{n p} \Phi_{p m}=\Phi_{n m}$ whenever $m \leq p \leq n$, where $T_{p_{n}}(K)$ is the $K$-algebra of all $p_{n} \times p_{n}$ upper triangular matrices over $K$. A triangular $U H F(T U H F) K$-algebra is any $K$-algebra that is $K$-isomorphic to an algebraic inductive limit of the form $\mathscr{T}=\underline{\lim }\left(T_{p_{n}}(K) ; \Phi_{n m}\right)$.

Definition 1.3. Let $\left(p_{n}\right)$ be a sequence of positive integers. The supernatural number of $\left(p_{n}\right)$ is the function $N\left[\left(p_{n}\right)\right]$, defined on the set of prime numbers, such that for any prime number $x$,

$$
N\left[\left(p_{n}\right)\right](x)=\sup \left\{m\left|\exists n: x^{m}\right| p_{n}\right\} .
$$

We are now ready to present a detailed statement of Theorem I and Lemma I.

Theorem I. Let $\mathscr{S}$ and $\mathscr{T}$ be TUHF $K$-algebras given by

$$
\mathscr{S}=\lim _{\longrightarrow}\left(T_{p_{n}}(K) ; \Phi_{n m}\right), \quad \mathscr{T}=\lim _{\longrightarrow}\left(T_{q_{n}}(K) ; \Psi_{n m}\right) .
$$

For each $n$, let $\left\{F_{i j}^{(n)} \mid 1 \leq i, j \leq q_{n}\right\}$ be the standard system of matrix units for $T_{q_{n}}(K)$. Assume that the following condition holds:

(i) For $m \leq n$,

$$
\operatorname{rank} \Psi_{n m}\left(F_{i j}^{(m)}\right)=\operatorname{rank} \Psi_{n m}\left(F_{u v}^{(m)}\right), \quad 1 \leq i \leq j \leq q_{m}, \quad 1 \leq u \leq v \leq q_{m} .
$$

If $\Phi: \mathscr{S} \rightarrow \mathscr{T}$ is a K-algebra isomorphism from $\mathscr{S}$ onto $\mathscr{T}$, then $N\left[\left(q_{n}\right)\right] \leq$ $N\left[\left(p_{n}\right)\right]$.

Moreover, for each $n$, let $\left\{E_{i j}^{(n)} \mid 1 \leq i, j \leq p_{n}\right\}$ be the standard system of matrix units for $T_{p_{n}}(K)$. If the following condition also holds, then $\mathscr{S}$ is $K$ isomorphic to $\mathscr{T}$, only if $N\left[\left(p_{n}\right)\right]=N\left[\left(q_{n}\right)\right]$ :

(ii) For $m \leq n$

$$
\operatorname{rank} \Phi_{n m}\left(E_{i j}^{(m)}\right)=\operatorname{rank} \Phi_{n m}\left(E_{u v}^{(m)}\right), \quad 1 \leq i \leq j \leq p_{m}, \quad 1 \leq u \leq v \leq p_{m} .
$$

The conclusion of Theorem I is strong in the sense that the TUHF $K$-algebra $\mathscr{S}$ is completely arbitrary; moreover, it is not assumed, a priori, that $\mathscr{S}$ or $\mathscr{T}$ have "diagonals" of any sort. Some condition similar to the above dimensionality condition (i) seems necessary. To see this, let $\left(p_{n}\right)$ and $\left(q_{n}\right)$ be any two unbounded, increasing sequences of positive integers. For $m \leq n$, define the mapping $\Phi_{n m}: M_{m}(K) \rightarrow M_{n}(K)$ by $\Phi_{n m}(x)=\operatorname{diag}\left(x, x_{m m}, \ldots, x_{m m}\right)$, $x=\left(x_{i j}\right) \in M_{m}(K)$. Then the TUHF $K$-algebras $\mathscr{S}=\underline{\lim }\left(T_{p_{n}}(K) ; \Phi_{p_{n} p_{m}}\right)$ and $\mathscr{T}=\lim \left(T_{q_{n}}(K) ; \Phi_{q_{n} q_{m}}\right)$ are isomorphic as $K$-algebras; but, clearly, there is not necessarily a nontrivial relation between $N\left[\left(p_{n}\right)\right]$ and $N\left[\left(q_{n}\right)\right]$. 
Lemma I. Let $n, p, q$ be positive integers. Let $\left\{G_{i j} \mid 1 \leq i \leq j \leq p\right\}$ be a triangular system of algebraic matrix units in $M_{n}(K)$. Let $\mathscr{A}$ be the subalgebra of $M_{n}(K)$ generated by the $G_{i j}$. Let $\left\{F_{i j} \mid 1 \leq i \leq j \leq q\right\}$ be a family of matrices in $\mathscr{A}$ such that the following conditions hold:

(i) $\left\{F_{i i} \mid 1 \leq i \leq q\right\}$ is an orthogonal family of idempotents such that $\sum_{i=1}^{q} F_{i i}$ $=1$;

(ii) for $1 \leq i \leq j \leq q, F_{i j}=F_{i i} F_{i j} F_{j j}$;

(iii) for $1 \leq i \leq j \leq q$ and $1 \leq r \leq s \leq q, \operatorname{rank}\left(F_{i j}\right)=\operatorname{rank}\left(F_{r s}\right)$.

Then $q \mid p$.

Theorem I can be derived from Lemma I in a few lines of simple reasoning. Thus, the bulk of the paper is devoted to the proof of Lemma I.

The proof of Lemma I has the virtue of being completely self-contained, and it is "elementary" in the sense that anyone with a knowledge of elementary matrix theory will be able to follow the proof.

Lemma I seems interesting in its own right-in fact, the author has used this lemma at a key point in [B2]. The lemma is a nontrivial "triangular" version of the fact that if $p, q$ are positive integers, then there exists a unital $K$-monomorphism $\Phi: M_{q}(K) \rightarrow M_{p}(K)$, only if $q \mid p$.

A natural question to ask is whether or not the converse of Theorem I holds: If two triangular UHF $K$-algebras have the same supernatural number, then are the algebras necessarily $K$-isomorphic to each other? The answer is "No." To see this, let $p, q$ be positive integers such that $q \mid p$ and define $d=p / q$. Define mappings $\rho_{p q}, \sigma_{p q}: M_{q}(K) \rightarrow M_{p}(K)$ by $\rho_{p q}(x)=x \otimes 1_{d}$, $\sigma_{p q}(x)=1_{d} \otimes x, x \in M_{q}(K)$. Note that $\rho_{p q}, \sigma_{p q}$ map $T_{q}(K)$ into $T_{p}(K)$. Now let $\left(p_{n}\right)$ be an unbounded sequence of positive integers such that $p_{m} \mid p_{n}$ whenever $m \leq n$. The refinement triangular UHF $K$-algebra determined by the sequence $\left(p_{n}\right)$ is the algebra $\mathscr{R}=\lim \left(T_{p_{n}}(K) ; \underline{\lim } \rho_{p_{n} p_{m}}\right)$, and the standard triangular UHF $K$-algebra determined by the sequence $\left(p_{n}\right)$ is the algebra $\Sigma=\underline{\lim }\left(T_{p_{n}}(K) ; \sigma_{p_{n} p_{m}}\right)$. The algebras $\mathscr{R}, \Sigma$ satisfy all conditions of Theorem I, and these algebras have the same supernatural number, but $\mathscr{R}$ is not $K$ isomorphic to $\Sigma$. To prove this, define $E \in T_{p_{1}}(K)$ by $E=\operatorname{diag}(1,0, \ldots, 0)$, and let $e$ be the canonical image of $E$ in $\mathscr{R}$. Then we have $(1-e) x e=0$ for all $x \in \mathscr{R}$. Now suppose that $\mathscr{R}$ is $K$-isomorphic to $\Sigma$ via $\Phi: \mathscr{R} \rightarrow \Sigma$. Then, with $f=\Phi(e)$, we have $(1-f) x f=0$ for all $x \in \Sigma$. But then $f$ lies in $\mathscr{D}=\bigcup \mathscr{D}_{n}$, where $\mathscr{D}_{n}$ is the canonical image of $D_{p_{n}}(K)$ in $\Sigma$. Here, $D_{p_{n}}(K)$ is the $K$-algebra of all $p_{n} \times p_{n}$ diagonal matrices over $K$. From this, it follows that $f=0$ or 1 . But this contradicts the fact that $f$ is nontrivial. Thus, $\mathscr{R}$ is not $K$-isomorphic to $\Sigma$.

There exist certain classes of triangular UHF $K$-algebras such that, within any of these classes, Theorem I and its converse hold. This is the content of the following theorem, which is a straightforward consequence of Theorem I: For each pair of positive integers $p, q$ such that $q \mid p$, let there correspond a unital $K$-monomorphism $\boldsymbol{\Theta}_{p q}: M_{q}(K) \rightarrow M_{p}(K)$ with the property that $\boldsymbol{\Theta}_{p r} \boldsymbol{\Theta}_{r q}=\boldsymbol{\Theta}_{p q}$ whenever $q|r| p$. Let $\left(p_{n}\right)$ and $\left(q_{n}\right)$ be sequences of positive integers such that $p_{m} \mid p_{n}$ and $q_{m} \mid q_{n}$ whenever $m \leq n$. Finally, let $\mathscr{S}$ and $\mathscr{T}$ be the TUHF $K$-algebras defined by $\mathscr{S}=\underline{\lim }\left(T_{p_{n}}(K) ; \boldsymbol{\Theta}_{p_{n} p_{m}}\right), \mathscr{T}=\underline{\lim }\left(T_{q_{n}}(K) ; \boldsymbol{\Theta}_{q_{n} q_{m}}\right)$. Then $\mathscr{S}$ is $K$-isomorphic to $\overrightarrow{\mathscr{T}}$ if, and only if $N\left[\left(p_{n}\right)\right]=N\left[\left(q_{n}\right)\right]$. This the- 
orem is a generalized, purely algebraic extension of the main result in [B1]. In particular, the theorem applies to the mappings $\rho_{p q}$ and $\sigma_{p q}$, i.e., two refinement TUHF $K$-algebras are $K$-isomorphic if and only if they have the same supernatural number-ditto for standard TUHF $K$-algebras.

Throughout the remainder of this paper, all algebras, monomorphisms, and isomorphisms will be assumed to be $K$-algebras, $K$-monomorphisms, and $K$ isomorphisms.

Historical remarks. In the 1990 paper [B1], the author introduced a concrete class of nonselfadjoint operator algebras which are currently called standard triangular $U H F(T U H F)$ algebras. The main result in [B1] is that two standard TUHF algebras are isometrically isomorphic if and only if they have the same supernatural number.

Standard triangular UHF algebras are special cases of what are called triangular UHF algebras. Triangular UHF algebras are defined as follows. Let $\mathscr{A}=\overline{U \mathscr{M}_{n}}$ be a UHF $C^{*}$-algebra, where $\left(\mathscr{M}_{n}\right)$ is an increasing sequence of finite-dimensional $C^{*}$-subalgebras of $\mathscr{A}$ such that for each $n, \mathscr{M}_{n}$ contains the identity of $\mathscr{A}$ and where $\mathscr{M}_{n}$ is *-isomorphic to $M_{p_{n}}$ for some positive integer $p_{n}$. A unital Banach algebra $\mathscr{T} \subseteq \mathscr{A}$ is said to be a triangular $U H F$ $(T U H F)$ algebra if $\mathscr{T}=\overline{\bigcup \mathscr{T}_{n}}$, where $\left(\mathscr{T}_{n}\right)$ is an increasing sequence of finitedimensional Banach subalgebras of $\mathscr{A}$ such that for each $n, \mathscr{T}_{n}$ is a Banach subalgebra of $\mathscr{M}_{n}$ and the diagonal, $\mathscr{T}_{n}{ }^{*} \cap \mathscr{T}_{n}$, of $\mathscr{T}_{n}$ is a maximal abelian selfadjoint subalgebra of $\mathscr{M}_{n}$. Standard TUHF algebras are those TUHF algebras such that the embedding of $\mathscr{M}_{n}$ into $\mathscr{M}_{n+1}$ has the special form

$$
x \mapsto 1 \otimes x .
$$

In [B2], the above definition of TUHF algebras is extended to the category of Banach algebras; these generalized TUHF algebras are called triangular UHF (TUHF) Banach algebras: A Banach algebra $\mathscr{T}$ is said to be a triangular UHF (TUHF) Banach algebra if $\mathscr{T}=\overline{\mathscr{T}_{n}}$, where $\left(\mathscr{T}_{n}\right)$ is an increasing sequence of finite-dimensional Banach subalgebras of $\mathscr{T}$ such that each $\mathscr{T}_{n}$ is generated, as a Banach algebra, by a triangular system of matrix units $\left\{e_{i j}^{(n)} \mid 1 \leq i \leq j \leq p_{n}\right\}$ in $\mathscr{T}$.

The idea of a TUHF $K$-algebra, where $K$ is an arbitrary field, arose from the author's desire to convert the principal result in [B2] into a pure piece of algebra (to borrow a phrase used by Kaplansky [AAR]). In fact, Theorem $I$ is a purely algebraic version of the main result in [B2]; and, as mentioned above, Theorem I can be used to derive a purely algebraic version of the main result in [B1].

We conclude our historical remarks by mentioning that the classification result in [B1] has been extended by Peters, Poon, and Wagner in [PPW] and by Power in [P1, P2].

This paper is organized as follows. In $\S 2$ we prove Lemma I in $\S 3$ we present the short proof of Theorem I.

\section{Proof of Lemma I}

In this section we prove a series of lemmas which culminates in the proof of Lemma I. The first lemma of this section is indispensable in the proof of Lemma I. 
Lemma 2.1. Let $n$ be a positive integer. Let $A, X, Y$ be matrices in $T_{n}(K)$. Suppose that $A=X A=A Y$. Then

$$
\operatorname{rank}(A)=\operatorname{rank}(\operatorname{diag}(X) A \operatorname{diag}(Y)) .
$$

Proof. Let $X_{1}=\operatorname{diag}(X), Y_{1}=\operatorname{diag}(Y)$, and $A_{1}=A Y_{1}$. We claim that $X_{1}$ is injective on the range of $A_{1}$, i.e., $\operatorname{rank}\left(X_{1} A_{1}\right)=\operatorname{rank}\left(A_{1}\right)$. To prove this, let $x \in K^{n}$ be such that $X_{1} A_{1} x=0$. Then, because $X A_{1}=A_{1}$, we have

$$
\left(X-X_{1}\right) A_{1} x=A_{1} x \text {. }
$$

The matrix $X-X_{1}$ is nilpotent, therefore there exists a smallest nonnegative integer $m$ such that $\left(X-X_{1}\right)^{m} A_{1} x=0$. We must have $m=0$; for if $m>0$, then, by (2), $\left(X-X_{1}\right)^{m-1} A_{1} x=\left(X-X_{1}\right)^{m} A_{1} x=0$, which is a contradiction. It follows that $A_{1} x=0$. This proves the claim. Thus, $\operatorname{rank}\left(X_{1} A_{1}\right)=\operatorname{rank}\left(A_{1}\right)$. Now, because $Y^{t} A^{t}=A^{t}$, we may apply the same argument to the pair $A^{t}$, $Y_{1}^{t} ;$ thus, $\operatorname{rank}(A)=\operatorname{rank}\left(A^{t}\right)=\operatorname{rank}\left(Y_{1}^{t} A^{t}\right)=\operatorname{rank}\left(A Y_{1}\right)$, and hence $\operatorname{rank}(A)=$ $\operatorname{rank}\left(X_{1} A Y_{1}\right)$.

The next lemma states that if $\left\{E_{i j} \mid 1 \leq i \leq j \leq p\right\}, E_{i j} \in M_{n_{i}, n_{j}}(K)$, are the blocks of a triangular system of algebraic matrix units in $M_{n}(K)$, where $n=\sum_{i=1}^{p} n_{i}$, then we can "diagonalize", in a certain way, block row 1 of this system; and Lemma 2.3 states that for arbitrary $1 \leq i \leq p$ we can diagonalize, in a similar way, block row $i$ of this system.

Lemma 2.2. Let $\left(n_{k}\right)_{k=1}^{p}$ be a sequence of positive integers. For $1 \leq i \leq j \leq p$, let $0 \neq E_{i j} \in M_{n_{i}, n_{j}}(K)$. Assume that the following conditions hold:

(i) for $1 \leq i \leq p, E_{i i}=1_{n_{i}}$;

(ii) for $1 \leq i \leq k \leq j \leq p, E_{i k} E_{k j}=E_{i j}$.

Then there exists invertible matrices $M_{1}, \ldots, M_{p}$ and a sequence $\left(d_{k}\right)_{k=1}^{p}$ of positive integers such that for $1 \leq j \leq p, M_{j} \in M_{n_{j}}(K)$ and

$$
n_{1}=d_{1} \geq d_{2} \geq \cdots \geq d_{p} ; \quad n_{j} \geq d_{j} ; \quad M_{1}^{-1} E_{1 j} M_{j}=\left(\begin{array}{cc}
1_{d_{j}} & 0 \\
0 & 0
\end{array}\right) .
$$

Proof. For $p=1$, take $M_{1}=1_{n_{1}}, d_{1}=n_{1}$, then $M_{1}^{-1} E_{11} M_{1}=1_{n_{1}}$. So the proof is trivial for $p=1$. Now suppose that the lemma is true for $p \geq 1$. Let $\left\{E_{i j} \mid 1 \leq i \leq j \leq p+1\right\}$ be a family of $n_{i} \times n_{j}$ matrices such that:

(iii) $E_{i i}=1_{n_{i}}, 1 \leq i \leq p+1$;

(iv) $E_{i k} E_{k j}=E_{i j}, 1 \leq i \leq k \leq j \leq p+1$.

The family $\left\{E_{i j} \mid 1 \leq i \leq j \leq p\right\}$ satisfies (i) and (ii); hence, by induction hypothesis, there exist invertible matrices $N_{j} \in M_{n_{j}}(K), 1 \leq j \leq p$, and a sequence $\left(d_{j}\right)_{j=1}^{p}$ of positive integers such that

$$
\begin{gathered}
n_{1}=d_{1} \geq d_{2} \geq \cdots \geq d_{p} ; \quad n_{j} \geq d_{j} ; \\
N_{1}^{-1} E_{1 j} N_{j}=\left(\begin{array}{cc}
1_{d_{j}} & 0 \\
0 & 0
\end{array}\right), \quad 1 \leq j \leq p .
\end{gathered}
$$

Define $N_{p+1}=1_{n_{p+1}}$, and let $F_{i j}, 1 \leq i \leq j \leq p+1$, be defined by

$$
F_{j p+1}=N_{j}^{-1} E_{j p+1}, \quad 1 \leq j \leq p+1 ; \quad F_{i j}=N_{i}^{-1} E_{i j} N_{j}, \quad 1 \leq i \leq j \leq p .
$$

Then we have

(v) $F_{i i}=1_{n_{i}}, 1 \leq j \leq p+1$;

(vi) $F_{i k} F_{k j}=F_{i j}, 1 \leq i \leq k \leq j \leq p+1$. 
Because $F_{p p+1}$ is an $n_{p} \times n_{p+1}$ matrix and because $d_{p} \leq n_{p}$, we can write $F_{p p+1}=\left(\begin{array}{c}X \\ Y\end{array}\right)$, where $X$ is a $d_{p} \times n_{p+1}$ matrix. We then have

$$
0 \neq F_{1 p+1}=F_{1 p} F_{p p+1}=N_{1}^{-1} E_{1 p} N_{p}\left(\begin{array}{c}
X \\
Y
\end{array}\right)=\left(\begin{array}{c}
X \\
0
\end{array}\right) \text {. }
$$

We conclude that $X \neq 0$. A result from matrix algebra [BL, Theorem 10.6] allows us to find a $d_{p} \times d_{p}$ matrix $M$ and an $n_{p+1} \times n_{p+1}$ matrix $N$ such that

$$
M^{-1} X N\left(\begin{array}{rr}
1_{d_{p+1}} & 0 \\
0 & 0
\end{array}\right), \quad d_{p+1}=\min \left\{d_{p}, n_{p+1}\right\} .
$$

Define $M_{i}, 1 \leq i \leq p+1$, by

$$
M_{p+1}=N ; \quad M_{i}=N_{i}\left(\begin{array}{cc}
M & 0 \\
0 & 1_{n_{i}-d_{p}}
\end{array}\right), \quad 1 \leq i \leq p .
$$

Note that the matrices $M_{i}$ are well defined, because, by (3), $n_{i} \geq d_{p}$ for $1 \leq$ $i \leq p$. For $1 \leq j \leq p$, we have

$$
\begin{aligned}
M_{1}^{-1} E_{1 j} M_{j} & =\left(\begin{array}{cc}
M^{-1} & 0 \\
0 & 1_{n_{1}-d_{p}}
\end{array}\right)\left(\begin{array}{ccc}
1_{d_{p}} & 0 & 0 \\
0 & 1_{d_{j}-d_{p}} & 0 \\
0 & 0 & 0
\end{array}\right)\left(\begin{array}{cc}
M & 0 \\
0 & 1_{n_{j}-d_{p}}
\end{array}\right) \\
& =\left(\begin{array}{cc}
1_{d_{j}} & 0 \\
0 & 0
\end{array}\right) ;
\end{aligned}
$$

here, we have used the fact that, by (3), $n_{1} \geq d_{j}$. By (4) and (5),

$$
M_{1}^{-1} E_{1 p+1} M_{p+1}=\left(\begin{array}{cc}
1_{d_{p+1}} & 0 \\
0 & 0
\end{array}\right) \text {. }
$$

Combining conditions (4)-(7), we see that for $1 \leq j \leq p+1$

$$
n_{1}=d_{1} \geq \cdots \geq d_{p+1} ; \quad n_{j} \geq d_{j} ; \quad M_{1}^{-1} E_{1 j} M_{j}=\left(\begin{array}{cc}
1_{d_{j}} & 0 \\
0 & 0
\end{array}\right) .
$$

This completes the inductive step from $p$ to $p+1$; hence, the lemma is proved.

Lemma 2.3. Let $\left(n_{i}\right)_{i=1}^{p}$ be a sequence of positive integers. Let $\left\{E_{i j} \mid 1 \leq i \leq j \leq\right.$ $p\}$ be a family of nonzero matrices over $K$ such that $E_{i j} \in M_{n_{i}, n_{j}}(K)$ and the $E_{i j}$ satisfy conditions (i) and (ii) of Lemma 2.2. Let $1 \leq i \leq p$ be arbitrary. Then there exist invertible matrices $N_{j} \in M_{n_{j}}(K), 1 \leq j \leq p$, and a sequence of positive integers $\left(m_{j}\right)_{j=1}^{p}$ such that for $1 \leq i \leq j \leq p$,

$$
n_{i}=m_{i} \geq m_{j} \geq m_{j+1} \geq \cdots \geq m_{p} ; \quad n_{j} \geq m_{j} ; \quad N_{i}^{-1} E_{i j} N_{j}=\left(\begin{array}{cc}
1_{m_{j}} & 0 \\
0 & 0
\end{array}\right) .
$$

Proof. For $1 \leq j \leq k \leq p-i+1$, define $n_{j}^{\prime}$ and $F_{j k} \neq 0$ by

$$
n_{j}^{\prime}=n_{i+(j-1)}, \quad F_{j k}=E_{i+(j-1), i+(k-1)} .
$$

Now apply Lemma 2.2 to the sequence $\left(n_{j}^{\prime}\right)_{j=1}^{p-i+1}$ and the family $\left\{F_{j k} \mid 1 \leq j \leq\right.$ $k \leq p-i+1\}$.

Lemmas 2.4 and 2.6 are technical lemmas whose proofs have been separated from the proof of Lemma I. Lemma 2.5, which is presented without proof, is a simple but useful result from matrix algebra. 
Lemma 2.4. Let $\left(n_{i}\right)_{i=1}^{p}$ be a sequence of positive integers. Let $\left\{E_{i j} \mid 1 \leq i \leq j \leq\right.$ $p$ \} be a family of nonzero matrices over $K$ such that $E_{i j} \in M_{n_{i}, n_{j}}(K)$. Assume that the following conditions hold:

(i) $E_{i i}=1_{n_{i}}, \quad 1 \leq i \leq p$;

(ii) $E_{i k} E_{k j}=E_{i j}, 1 \leq i \leq k \leq j \leq p$.

Define $n=\sum_{i=1}^{p} n_{i}$. For $1 \leq i, j \leq p$, let $G_{i j} \in T_{n}(K)$ be the block upper triangular matrix such that for $1 \leq d \leq k \leq p$, the $d-k$ block entry is $0_{n_{d} \times n_{k}}$, if $(d, k) \neq(i, j)$, and the $i$-j entry of $G_{i j}$ is $E_{i j}$. Let $0 \neq H \in M_{n}(K)$ have the form

$$
H=\sum_{1 \leq i \leq j}^{p} h_{i j} G_{i j}
$$

and suppose that the following conditions hold:

(iii) For $1 \leq i \leq p$, define $G_{i}=G_{i i}$. There exist sequences of positive integers

$$
1 \leq r_{1}<\cdots<r_{d} \leq p ; \quad 1 \leq s_{1}<\cdots<s_{m} \leq p
$$

such that $E F=0$ and $H=E H=H F$, where

$$
E=\sum_{k=1}^{d} G_{r_{k}}, \quad F=\sum_{k=1}^{m} G_{s_{k}} .
$$

(iv) For some pair $1 \leq u \leq d, 1 \leq v \leq m$ of positive integers, $r_{u} \leq s_{v}$ and ihe matrix $h_{r_{u} s_{v}} E_{r_{u} s_{v}}$ is the first nonzero block entry in block row $r_{u}$ and the last nonzero block entry in block column $s_{v}$.

(v) $\operatorname{rank}(H)=\sum_{k=1}^{d} n_{r_{k}}=\sum_{k=1}^{m} n_{s_{k}}$, where $n_{i}=\operatorname{dim}\left(G_{i}\right), \quad 1 \leq i \leq p$.

Then there exists a matrix $J \in T_{n}(K)$ of the form $J=\sum_{1 \leq i \leq j}^{p} g_{i j} G_{i j}$ such that the following conditions are satisfied:

(vi) $J=E J=J F$;

(vii) the matrix $g_{r_{u} s_{v}} E_{r_{u} s_{v}}$ is the only nonzero block entry in block row $r_{u}$ and the only nonzero block entry in block column $s_{v}$;

(viii) $\operatorname{rank}(J)=\operatorname{rank}(H)$.

Proof. By Lemma 2.3, there exist invertible matrices $N_{j} \in M_{n_{j}}(K), 1 \leq j \leq p$, and a sequence of positive integers $\left(q_{j}\right)_{j=r_{u}}^{p}$ such that for $r_{u} \leq j \leq p$,

$$
n_{r_{u}}=q_{r_{u}} \geq q_{j} \geq q_{j+1} \geq \cdots \geq q_{p} ; \quad n_{j} \geq q_{j} ; \quad N_{r_{u}}^{-1} E_{r_{u} j} N_{j}=\left(\begin{array}{cc}
1_{q_{j}} & 0 \\
0 & 0
\end{array}\right) .
$$

Define $N \in M_{n}(K)$ by $N=\operatorname{diag}\left(N_{1}, \ldots, N_{p}\right)$. For $1 \leq i \leq j \leq p$, define $F_{i j}, H_{i j}$ by

$$
F_{i j}=N_{i}^{-1} E_{i j} N_{j}, \quad H_{i j}=N^{-1} G_{i j} N ;
$$

then it is easy to see that for $1 \leq d, k \leq p$, the $d-k$ block entry of $H_{i j}$ is $0_{n_{d} n_{k}}$, if $(d, k) \neq(i j)$, and the $i$-j block entry of $H_{i j}$ is $F_{i j}$; so, $H_{i j} \in T_{n}(K)$. Define the matrices $L, P, Q \in T_{n}(K)$ by

$$
L=N^{-1} H N=\sum_{1 \leq i \leq j}^{p} h_{i j} H_{i j}, \quad P=N^{-1} E N, \quad Q=N^{-1} F N .
$$

We then have

(ix) $L=P L=L Q$; 
(x) $F_{i i}=1_{n_{i}}, 1 \leq i \leq p$;

(xi) $F_{i k} F_{k j}=F_{i j}, 1 \leq i \leq k \leq j \leq p$;

(xii) $h_{r_{u} s_{v}} F_{r_{u} s_{v}}$ is the first nonzero block entry in block row $r_{u}$ and the last nonzero block entry in block column $s_{v}$;

(xiii) $\operatorname{rank}(L)=\operatorname{rank}(H)$.

Let $1 \leq i<r_{u}$ and $s_{v}<j \leq p$, and write

$$
F_{i r_{u}}=\left(\begin{array}{ccc}
a_{11}^{(i)} & \cdots & a_{1 n_{r_{u}}}^{(i)} \\
\vdots & & \vdots \\
a_{n_{i} 1}^{(i)} & \cdots & a_{n_{i} n_{r_{u}}}^{(i)}
\end{array}\right)
$$

by (8), $n_{r_{u}}=q_{r_{u}} \geq q_{s_{v}} \geq q_{j}$, consequently,

$$
\begin{aligned}
F_{i j}=F_{i r_{u} F_{r_{u} j}} & =\left(\begin{array}{ccc}
a_{11}^{(i)} & \cdots & a_{1 n_{r_{u}}}^{(i)} \\
\vdots & & \vdots \\
a_{n_{i 1}}^{(i)} & \cdots & a_{n_{i} n_{r_{u}}}^{(i)}
\end{array}\right)\left(\begin{array}{cccc}
1_{q_{j}} & 0 \\
0 & 0
\end{array}\right) \\
& =\left(\begin{array}{cccccc}
a_{11}^{(i)} & \cdots & a_{1 q_{j}}^{(i)} & 0 & \cdots & 0 \\
\vdots & & \vdots & \vdots & & \vdots \\
a_{n_{i 1}}^{(i)} & \cdots & a_{n_{i} q_{j}}^{(i)} & 0 & \cdots & 0
\end{array}\right) .
\end{aligned}
$$

It follows from (9) that for $1 \leq i<r_{u}$ and $s_{v}<j \leq p$, the entries $\begin{gathered}\left(i, s_{v}\right) \\ \left(r_{u}, s_{v}\right)\end{gathered}\left(\begin{array}{l}(i, j) \\ \left(r_{u}, j\right)\end{array}\right.$ of $L$ can be expressed as follows:

(10)

$$
\begin{array}{ll}
h_{i s_{v}}\left(\begin{array}{cccccc}
a_{11}^{(i)} & \cdots & a_{1 q_{s_{v}}}^{(i)} & 0 & \cdots & 0 \\
\vdots & & \vdots & \vdots & & \vdots \\
a_{n_{i} 1}^{(i)} & \cdots & a_{n_{i} q_{s_{v}}}^{(i)} & 0 & \cdots & 0
\end{array}\right), h_{i j}\left(\begin{array}{cccccc}
a_{11}^{(i)} & \cdots & a_{1 q_{j}}^{(i)} & 0 & \cdots & 0 \\
\vdots & & \vdots & \vdots & & \vdots \\
a_{n_{i} 1}^{(i)} & \cdots & a_{n_{i} q_{j}}^{(i)} & 0 & \cdots & 0
\end{array}\right), \\
h_{r_{u} s_{v}}\left(\begin{array}{cc}
1_{q_{s v}} & 0 \\
0 & 0
\end{array}\right), & h_{r_{u} j}\left(\begin{array}{cc}
1_{q_{j}} & 0 \\
0 & 0
\end{array}\right) .
\end{array}
$$

Because $h_{r_{u}} s_{v} F_{r_{u}} s_{v}$ is the first nonzero block entry in block row $r_{u}$, and because $1 \leq i<r_{u}$ and $s_{v}<j \leq p$ are arbitrary, it follows from (xii) and (10) that by performing elementary row operations on $L$ we can transform $L$ into a matrix of the form $L^{\prime}=\sum_{1 \leq d \leq k}^{p} h_{d k}^{\prime} H_{d k}$ which has the property that $h_{r_{u} s_{v}}^{\prime} F_{r_{u} s_{v}}$ is the first nonzero block entry in block row $r_{u}$ and the only nonzero block entry in block column $s_{v}$, and $L^{\prime}=P L^{\prime}=L^{\prime} Q$. Because $L^{\prime}$ is row equivalent to $L$, it follows from (xiii) that

$$
\operatorname{rank}\left(L^{\prime}\right)=\operatorname{rank}(H)
$$

By (9), if $1 \leq i<r_{u}$ and $s_{v}<j \leq p$, then the entries $\underset{\left(r_{u}, s_{v}\right)\left(r_{u}, j\right)}{\left(i, s_{0}\right)}$ of $L^{\prime}$ can be expressed as follows: 
(12)

$$
\begin{aligned}
h_{i s_{v}}^{\prime}\left(\begin{array}{cccccc}
a_{11}^{(i)} & \cdots & a_{1}^{(i)} q_{s_{v}} & 0 & \cdots & 0 \\
\vdots & & \vdots & \vdots & & \vdots \\
a_{n_{i} 1}^{(i)} & \cdots & a_{n_{i} q_{s_{v}}}^{(i)} & 0 & \cdots & 0
\end{array}\right), & h_{i j}^{\prime}\left(\begin{array}{cccccc}
a_{1}^{(i)} & \cdots & a_{1 q_{j}}^{(i)} & 0 & \cdots & 0 \\
\vdots & & \vdots & \vdots & & \vdots \\
a_{n_{i} 1}^{(i)} & \cdots & a_{n_{i} q_{j}}^{(i)} & 0 & \cdots & 0
\end{array}\right), \\
h_{r_{u} s_{v}}^{\prime}\left(\begin{array}{cc}
1_{q_{s_{v}}} & 0 \\
0 & 0
\end{array}\right), & h_{r_{u j}}^{\prime}\left(\begin{array}{cc}
1_{q_{j}} & 0 \\
0 & 0
\end{array}\right) .
\end{aligned}
$$

For $s_{v}<j \leq p,(8)$ implies that $q_{j} \leq q_{s_{v}}$; therefore, because $h_{r_{u} s_{v}}^{\prime} F_{r_{u}} s_{v}$ is the first nonzero block entry in block row $r_{u}$ and the only nonzero block entry in block column $s_{v}$, and $L^{\prime}=P L^{\prime}=L^{\prime} Q$, it follows from (12) that by performing elementary column operations on $L^{\prime}$ we can transform $L^{\prime}$ into a matrix of the form $M=\sum_{1 \leq i \leq j}^{p} q_{i j} H_{i j}$ which has the property that $g_{r_{u} s_{v}} F_{r_{u} s_{v}}$ is the only nonzero block entry in block row $r_{u}$ and the only nonzero block entry in block column $s_{v}$, and $M=P M=M Q$. Because $M$ is column equivalent to $L^{\prime}$, (11) implies that

$$
\operatorname{rank}(M)=\operatorname{rank}(H) .
$$

Now define $J=N M N^{-1}=\sum_{1 \leq i \leq j}^{p} g_{i j} G_{i j}$. Then $J$ satisfies conditions (vi) and (vii) above; moreover, (13) implies that $J$ satisfies condition (viii). Hence we have completed the proof of the lemma.

Lemma 2.5. Let $i, j, n$ be positive integers. Let $X, Y, Z \in M_{n}(K)$. Suppose that

$$
X=Y+Z, \quad \operatorname{rank}(X)=i+j, \quad \operatorname{rank}(Y) \leq i, \quad \operatorname{rank}(Z) \leq j .
$$

Then $\operatorname{rank}(Y)=i$ and $\operatorname{rank}(Z)=j$.

Lemma 2.6. Let $\left(n_{i}\right)_{i=1}^{p}$ be a sequence of positive integers. Let $\left\{E_{i j} \mid 1 \leq i \leq j \leq\right.$ $p\}$ be a family of nonzero matrices over $K$ such that $E_{i j} \in M_{n_{i}, n_{j}}(K)$. Assume that the following conditions hold:

(i) $E_{i i}=1_{n_{i}}, 1 \leq i \leq p$;

(ii) $E_{i k} E_{k j}=E_{i j}, \quad 1 \leq i \leq k \leq j \leq p$.

Define $n=\sum_{i=1}^{p} n_{i}$. For $1 \leq i, j \leq p$, let $G_{i j} \in T_{n}(K)$ be the block upper triangular matrix defined in Lemma 2.4. Assume that there exists $0 \neq H \in$ $M_{n}(K)$ of the form $H=\sum_{1 \leq i \leq j}^{p} h_{i j} G_{i j}$ with the following properties:

(iii) There exist sequences of positive integers,

$$
1 \leq r_{1}<\cdots<r_{d} \leq p, \quad 1 \leq s_{1}<\cdots<s_{m} \leq p,
$$

such that $E F=0$ and $H=E H=H F$, where $E=\sum_{k=1}^{d} G_{r_{k}}, F=\sum_{k=1}^{m} G_{s_{k}}$;

(iv) $\operatorname{rank}(H)=\sum_{k=1}^{d} n_{r_{k}}=\sum_{k=1}^{m} n_{s_{k}}$.

Then $d=m$.

Proof. We will prove the lemma by induction on $d$. Assume, first, that $d=1$. By (iii), we have

$$
H=E H=G_{r_{1}} H=G_{r_{1}} \sum_{1 \leq i \leq j}^{p} h_{i j} G_{i j}=\sum_{j \geq r_{1}}^{p} h_{r_{1}} G_{r_{1} j}=\sum_{\substack{1 \leq k \leq m \\ s_{k} \geq r_{1}}} G_{r_{1}} s_{k} .
$$


Because $H \neq 0$, there is a smallest positive integer $1 \leq v \leq m$ such that $s_{v} \geq r_{1}$ and $h_{r_{1}} s_{v} \neq 0$; we then have

$$
H=\sum_{j=s_{v}}^{p} h_{r_{1} j} G_{r_{1}, j}
$$

For $s_{v} \leq j \leq p$, we have

$$
h_{r_{1} j} G_{r_{1} j}=\left(h_{r_{1} s_{v}}^{-1} h_{r_{1} j}\right)\left(h_{r_{1} s_{v}} G_{r_{1} s_{v}}\right) G_{s_{v} j} ;
$$

hence, $h_{r_{1} j} G_{r_{1} j}$ can be obtained from $h_{r_{1} s_{v}} G_{r_{1}} s_{v}$ by elementary column operations; therefore, $H$ is column equivalent to $J=h_{r_{1} s_{v}} G_{r_{1}} s_{v}$, so $\operatorname{rank}(H)=$ $\operatorname{rank}(J)$. From the definition of $G_{r_{1}} s_{v}$, we have

$$
\operatorname{rank}\left(h_{r_{1} s_{v}} G_{r_{1} s_{v}}\right)=\operatorname{rank}\left(E_{r_{1} s_{v}}\right) \leq n_{s_{v}} .
$$

Therefore, by (iv), $\operatorname{rank}(H)=\sum_{k=1}^{m} n_{s_{k}} \leq n_{s_{v}}$, from which it follows that $m=1=d$. This proves the lemma for the case when $d=1$.

Now suppose that the lemma is true for $d>1$. Thus, assume that we are given a matrix $0 \neq H=\sum_{1 \leq i \leq j}^{p} h_{i j} G_{i j}$ with the following properties:

(v) There exist sequences of positive integers,

$$
1 \leq r_{1}<\cdots<r_{d+1} \leq p, \quad 1 \leq s_{1}<\cdots<s_{m} \leq p,
$$

such that $E F=0$ and $H=E H=H F$, where $E=\sum_{k=1}^{d+1} G_{r_{k}}, F=\sum_{k=1}^{m} G_{s_{k}}$;

(vi) $\operatorname{rank}(H)=\sum_{k=1}^{d+1} n_{r_{k}}=\sum_{k=1}^{m} n_{s_{k}}$.

Because $H \neq 0$, there exist $1 \leq u \leq d+1$ and $1 \leq v \leq m$ such that $r_{u} \leq s_{v}$ and $h_{r_{u} s_{v}} E_{r_{u} s_{v}}$ is the first nonzero block entry in block row $r_{u}$ and the last nonzero block entry in block column $s_{v}$; hence, by (v) and (vi), we may apply Lemma 2.4 to obtain a matrix $J \in T_{n}(K)$ of the form $J=\sum_{1 \leq i \leq j}^{p} g_{i j} G_{i j}$ which has the following properties:

(vii) The matrix $g_{r_{u} s_{v}} E_{r_{u} s_{v}}$ is the only nonzero block entry in block row $r_{u}$ and the only nonzero block entry in block column $s_{v}$;

(viii) $J=E J=J F$;

(ix) $\operatorname{rank}(J)=\sum_{k=1}^{d+1} n_{r_{k}}=\sum_{k=1}^{m} n_{s_{k}}$.

By (vii), $G_{r_{u}} J=J G_{s_{v}}=g_{r_{u} s_{v}} G_{r_{u} s_{v}}$. Therefore, by (viii),

$$
J=E J F=\left(\sum_{k \neq u}^{d+1} G_{r_{k}}+G_{r_{u}}\right) J\left(\sum_{k \neq v}^{m} G_{s_{k}}+G_{s_{v}}\right)=J_{1}+J_{2} ;
$$

where $J_{1}=\left(\sum_{k \neq u}^{d+1} G_{r_{k}}\right) J\left(\sum_{k \neq v}^{m} G_{s_{k}}\right)$ and $J_{2}=g_{r_{u} s_{v}} G_{r_{u} s_{v}}$. Now, by (ix), we have

$$
\begin{gathered}
J=J_{1}+J_{2}, \\
\operatorname{rank}(J)=\sum_{k \neq u}^{d+1} n_{r_{k}}+n_{r_{u}}, \quad \operatorname{rank}\left(J_{1}\right) \leq \sum_{k \neq u}^{d+1} n_{r_{k}}, \operatorname{rank}\left(J_{2}\right)=\operatorname{rank}\left(G_{r_{u}} s_{v}\right) \leq n_{r_{u}} ;
\end{gathered}
$$


therefore, by Lemma 2.5, $\operatorname{rank}\left(J_{1}\right)=\sum_{k \neq u}^{d+1} n_{r_{k}}$. Likewise, $\operatorname{rank}\left(J_{1}\right)=\sum_{k \neq v}^{m} n_{s_{k}}$. The matrix $J_{1}$ clearly has the form $J_{1}=\sum_{1 \leq i \leq j}^{p} f_{i j} G_{i j}$. Define $E_{1}=\sum_{k \neq u}^{d+1} G_{r_{k}}$, $F_{1}=\sum_{k \neq v}^{m} G_{s_{k}}$. Then

$$
\begin{gathered}
J_{1}=\sum_{1 \leq i \leq j}^{p} f_{i j} G_{i j}, \\
E_{1} F_{1}=0, \quad J_{1}=E_{1} J_{1}=J_{1} F_{1}, \\
\operatorname{rank}\left(J_{1}\right)=\sum_{k \neq u}^{d+1} n_{r_{k}}=\sum_{k \neq v}^{m} n_{s_{k}}, \\
1 \leq r_{1}<\cdots<r_{u-1}<r_{u+1}<\cdots<r_{d+1} \leq p, \\
1 \leq s_{1}<\cdots<s_{v-1}<s_{v+1}<\cdots<s_{m} \leq p .
\end{gathered}
$$

Now apply the induction hypothesis to (14) to conclude that $d=m-1$, i.e., $d+1=m$. This completes the proof of the lemma.

We now have enough machinery to prove the main lemma of this section.

Proof of Lemma I. Let $n, p, q$ be positive integers, and let $\left\{G_{i j} \mid 1 \leq i \leq j \leq\right.$ $p\}, \mathscr{A}$, and $\left\{F_{i j} \mid 1 \leq i \leq j \leq q\right\}$ be as in the hypothesis of Lemma I. Lemma $\mathrm{I}$ is clearly true for $p=1$, so we may assume that $p>1$. For $1 \leq i \leq p$, define $G_{i}=G_{i i}, n_{i}=\operatorname{dim}\left(G_{i}\right)$. By simultaneously diagonalizing the family $\left\{G_{i i} \mid 1 \leq i \leq p\right\}$, we may assume, without loss of generality, that each $G_{i}$ has the form

$$
G_{i}=\operatorname{diag}\left(0, \ldots, 0,1_{n_{i}}, 0, \ldots, 0\right),
$$

where the matrix $1_{n_{i}}$ is the $i$ th block diagonal entry of $G_{i}$. Then there exists a family $\left\{H_{i j} \mid 1 \leq i \leq j \leq p\right\}$ of matrices over $K$ such that for $1 \leq i \leq j \leq p$, $H_{i j} \in M_{n_{i}, n_{j}}(K)$, and such that the following conditions hold:

(i) $H_{i i}=1_{n_{i}}, 1 \leq i \leq p$;

(ii) $H_{i k} H_{k j}=H_{i j}, 1 \leq i \leq k \leq j \leq p$;

(iii) for $1 \leq i \leq j \leq \bar{p}, G_{i j}$ is the block upper triangular matrix in $T_{n}(K)$ such that for $1 \leq \bar{d}, k \leq p$, the $d-k$ entry of $G_{i j}$ is $0_{n_{d} \times n_{k}}$, if $(d, k) \neq(i, j)$, and the $i-j$ entry of $G_{i j}$ is $H_{i j}$.

For $1 \leq i \leq q$, define $F_{i}=F_{i i}$; then because $F_{i}$ is an idempotent in $\mathscr{A}$, it is easy to see that there corresponds to $i$ a subsequence $\left(r_{i k}\right)_{k=1}^{m_{i}}$ of $1,2, \ldots, p$ such that

$$
\operatorname{diag}\left(F_{i}\right)=\sum_{k=1}^{m_{i}} G_{r_{i k}} .
$$

Fix $1 \leq i<j \leq q$. We have $F_{i}, F_{j}, F_{i j} \in T_{n}(K)$, with $F_{i j}=F_{i} F_{i j} F_{j}$; hence, by Lemma 2.1 ,

$$
\operatorname{rank}\left(F_{i j}\right)=\operatorname{rank}\left(\operatorname{diag}\left(F_{i}\right) F_{i j} \operatorname{diag}\left(F_{j}\right)\right) .
$$

Define $E=\operatorname{diag}\left(F_{i}\right), F=\operatorname{diag}\left(F_{j}\right)$, and $H=\operatorname{diag}\left(F_{i}\right) F_{i j} \operatorname{diag}\left(F_{j}\right)$. The matrix $H$ clearly has the form $\sum_{1<d<k}^{p} h_{d_{k}} G_{d k}$. Then $E F=0$ and $H=E H=H F$. By (15), $\operatorname{rank}(H)=\operatorname{rank}\left(\bar{F}_{i j}\right)=\operatorname{dim}\left(F_{i}\right)=\operatorname{dim}\left(F_{j}\right)$. We conclude that for 
$1 \leq i \leq j \leq p$

$$
\begin{gathered}
H=\sum_{1 \leq d \leq k}^{p} h_{d k} G_{d k}, \\
E F=0, \quad H=E H=H F, \\
\operatorname{rank}(H)=\sum_{k=1}^{m_{i}} n_{r_{i k}}=\sum_{k=1}^{m_{j}} n_{r_{j k}}, \\
1 \leq r_{i 1}<\cdots<r_{i m_{i}} \leq p, \\
1 \leq r_{j 1}<\cdots<r_{j m_{j}} \leq p .
\end{gathered}
$$

Conditions (i)-(iii) above allow us to apply Lemma 2.6 to (16), and we conclude that $m_{i}=m_{j}$. Because $1 \leq i<j \leq q$ are arbitrary, we have that for some positive integer $d, d=m_{i}$ for $1 \leq i \leq q$. Therefore,

$$
1=\sum_{i=1}^{p} G_{i}=\sum_{i=1}^{q} \operatorname{diag}\left(F_{i}\right)=\sum_{i=1}^{q}\left(\sum_{k=1}^{m_{i}} G_{r_{i k}}\right)=\sum_{i=1}^{q}\left(\sum_{k=1}^{d} G_{r_{i k}}\right) .
$$

It follows that $p=d q$. This completes the proof of the lemma.

\section{Proof of Theorem I}

In this final section we prove Theorem $\mathrm{I}$.

Proof of Theorem I. Let $\mathscr{S}=\underline{\lim }\left(T_{p_{n}}(K) ; \Phi_{n m}\right)$ and $\mathscr{T}=\underline{\lim }\left(T_{q_{n}}(K) ; \Psi_{n m}\right)$ be the TUHF $K$-algebras given in Theorem I. Assume that the mappings $\Psi_{n m}$ satisfy condition (i) of Theorem I. Suppose that $\Phi: \mathscr{S} \rightarrow \mathscr{T}$ is an isomorphism from $\mathscr{S}$ onto $\mathscr{T}$. We want to show that $N\left[\left(q_{n}\right)\right] \leq N\left[\left(p_{n}\right)\right]$. To prove this, it will suffice to show that for each $m$, there exists an $n$ such that $q_{m} \mid p_{n}$. So fix $m$. For each $n$, let $\Phi_{n}$ be the canonical embedding of $T_{p_{n}}(K)$ into $\mathscr{S}$ and let $\Psi_{n}$ be the canonical embedding of $T_{q_{n}}(K)$ into $\mathscr{T}$. Then $\mathscr{S}=\bigcup \mathscr{S}_{n}$ and $\mathscr{T}=\bigcup \mathscr{T}_{n}$. We have $\mathscr{T}=\bigcup \Phi\left(\mathscr{S}_{n}\right)$, hence, there exists an $n$ such that $\mathscr{T}_{m} \subseteq \Phi\left(\mathscr{S}_{n}\right)$. Because $\mathscr{T}=\bigcup \mathscr{T}_{k}$, there exists a $k \geq m$ such that $\Phi\left(\mathscr{S}_{n}\right) \subseteq \mathscr{T}_{k}$. For $1 \leq i \leq j \leq q_{m}$, define $f_{i j}=\Psi_{k m}\left(F_{i j}^{(m)}\right)$. For $1 \leq i \leq j \leq p_{n}$, define $h_{i j}=\Phi\left(\Phi_{n}\left(E_{i j}^{(n)}\right)\right), \quad g_{i j}=\Psi_{k}^{-1}\left(h_{i j}\right) \in T_{q_{k}}(K)$. Let $\mathscr{A}$ be the subalgebra of $M_{q_{k}}(K)$ generated by $g_{i j}, 1 \leq i \leq j \leq p_{n}$. Then we have $f_{i j} \in \mathscr{A}$ for $1 \leq i \leq j \leq q_{m}$. By assumption, we have

$$
\operatorname{rank}\left(f_{i j}\right)=\operatorname{rank}\left(f_{u v}\right), \quad 1 \leq i \leq j \leq q_{m}, \quad 1 \leq u \leq v \leq q_{m} .
$$

The families

$$
\left\{f_{i j} \mid 1 \leq i \leq j \leq q_{m}\right\}, \quad\left\{g_{i j} \mid 1 \leq i \leq j \leq p_{n}\right\}
$$

are triangular systems of algebraic matrix units in $M_{q_{k}}(K)$; therefore, by (17) and Lemma $\mathrm{I}$, we conclude that $q_{m} \mid p_{n}$. Because $m$ is arbitrary, this proves the first part of Theorem I. The last part of Theorem I is an immediate consequence of the first part. 


\section{REFERENCES}

[AAR] D. J. Alkers, G. L. Alexanderson, asnd C. Reid, More mathematical people, Harcourt Brace Jovanovich, 1990.

[B1] R. Baker, Triangular UHF algebras, J. Funct. Anal. 91 (1990), 182-212.

[B2] - On certain Banach limits of triangular matrix algebras, Houston J. Math. (to appear).

[BL] T. S. Blyth, Module theory, Oxford Univ. Press, London, 1990.

[PPW] J. R. Peters, Y. T. Poon, and B. H. Wagner, Triangular AF algebras, Proc. J. Operator Theory 23 (1990), 81-114.

[P1] S. C. Power, Classifications of tensor products of triangular matrix algebras, Proc. London Math. Soc. (3) 61 (1990), 571-614.

[P2] - The classification of triangular subalgebras of $C^{*}$-algebras, Bull London Math. Soc. 22 (1990), 264-272.

Department of Mathematics, University of Iowa, Iowa City, Iowa 52242

E-mail address: baker@math.uiowa.edu 